\title{
Meta-design: Expanding Boundaries and Redistributing Control in Design
}

\author{
Gerhard Fischer \\ Center for Lifelong Learning and Design \\ University of Colorado, Boulder \\ http: //13d.cs.colorado.edu/ gerhard/
}

\begin{abstract}
Meta-design is an emerging conceptual framework aimed at defining and creating socio-technical environments as living entities. It extends existing design methodologies focused on the development of a system at design time by allowing users to become co-designers at use time. Meta-design is grounded in the basic assumption that future uses and problems cannot be completely anticipated at design time, when a system is developed. Users, at use time, will discover mismatches between their needs and the support that an existing system can provide for them. Meta-design extends boundaries by supporting users as active contributors who can transcend the functionality and content of existing systems. By facilitating these possibilities, control is distributed among all stakeholders in the design process.

This paper characterizes different design methodologies and identifies the unique challenges and opportunities for meta-design. It illustrates this approach with two examples: (a) Web2Gether (enriching the organizational practices and community building of assistive technology teachers), and (b) the Memory Aiding Prompting System (MAPS) (addressing the needs of people with cognitive disabilities and their caregivers). Assessments of our developments are used to identify some future implications and challenges for meta-design and its role in socially responsible design.
\end{abstract}

Keywords: design, design methodologies, meta-design, socio-technical environments, boundaries, control, seeding / evolutionary growth / reseeding model, Web2Gether, Memory Aiding Prompting System (MAPS), application areas for meta-design, socially responsible design.

\section{Introduction}

In past decades, the primary goal of most software systems has been to achieve better productivity and usability, and software design and human-computer interface (HCI) research have achieved considerable expertise for these objectives. However, we have entered a new phase of system development in exploring new application areas (including the two examples discussed in this paper) with a focus on transcending existing boundaries and redistributing control among stakeholders [NationalResearch-Council, 2003]. Prominent success examples of this approach include developments such as (1) open source software [Raymond \& Young, 2001]; (2) collaborative developed encyclopedias such as Wikipedia [Wikipedia, 2006]; and (3) 
digital libraries such as DLESE [Wright et al., 2002]. In these developments people are not only using software but they also are becoming involved in developing software to varying degrees [Scaffidi et al., 2005]. Existing design methodologies are insufficient to cope with the emergence of situated and unintended requirements [Suchman, 1987; Winograd \& Flores, 1986]. What is needed are socio-technical environments for which the design does not end at the time of deployment and whose success hinges on continued user participation.

This paper addresses the overall theme of Interact'2007, Socially Responsible Interaction, by articulating meta-design as a new conceptual framework and by relating and contrasting it to existing design methodologies. The framework is instantiated and illustrated by a brief description of socio-technical environments in two different domains:

- enriching the organizational practices of assistive technology teachers with Web2Gether [dePaula, 2004]; and

- addressing the needs of new user populations, namely people with cognitive disabilities and their caregivers with the Memory Aiding Prompting System (MAPS) [Carmien, 2006].

\section{Meta-design: A Conceptual Framework and Design Methodology for Socio-technical Environments}

Meta-design [Fischer \& Giaccardi, 2006; Fischer et al., 2004; Giaccardi, 2004] is an emerging conceptual framework aimed at defining and creating social and technical infrastructures in which new forms of collaborative design can take place. It extends the traditional notion of system design beyond the original development of a system by allowing users to become co-designers. Meta-design is grounded in the basic assumption that future uses and problems cannot be completely anticipated at design time, when a system is developed. Users, at use time, will discover mismatches between their needs and the support that an existing system can provide for them. These mismatches will lead to breakdowns that serve as potential sources of new insights, new knowledge, and new understanding.

\subsection{Socio-technical Environments as Living Entities}

Socio-technical environments [Mumford, 1987; Trist, 1981] are living entities [Terveen, 1995] that are capable of integrating computing infrastructures and participation processes supporting collaboration not only about design artifacts but also about the goals of the design activity. By allowing users to be designers, sociotechnical environments offer the possibility to achieve the best fit between systems and their ever-changing context of use, problems, domains, users, and communities of users. They empower users, as owners of a problem, to engage actively and collaboratively in the continual development of systems capable of sustaining personally meaningful activities and coping with their emergent needs. Sociotechnical environments evolve as a result of a flexible and collaborative development process, which in turn modifies the terms of participation itself in the production of software. 
The rationale for socio-technical environments as living entities expanding boundaries and redistributing control in design comes from many sources, including the following prescriptive objectives and empirical observations:

- "The experience of having participated in a problem makes a difference to those who are affected by the solution. People are more likely to like a solution if they have been involved in its generation; even though it might not make sense otherwise" [Rittel, 1984].

- "I believe passionately in the idea that people should design buildings for themselves. In other words, not only that they should be involved in the buildings that are for them but that they should actually help design them" [Alexander, 1984].

- "We have only scratched the surface of what would be possible if end users could freely program their own applications. As has been shown time and again, no matter how much designers and programmers try to anticipate and provide for what users will need, the effort always falls short because it is impossible to know in advance what may be needed. End users should have the ability to create customizations, extensions, and applications" [Nardi, 1993].

- "The hacker culture and its successes pose by example some fundamental questions about human motivation, the organization of work, the future of professionalism, and the shape of the firm" [Raymond \& Young, 2001].

- "Users that innovate can develop exactly what they want, rather than relying on manufacturers to act as their (often very imperfect) agents" [von Hippel, 2005].

- "In the digital world, many of the distinctions between designers and users are becoming blurred. We are all, to some extent, designers now” [Brown \& Duguid, 2000a].

- "The networked environment makes possible a new modality of organizing production: radically decentralized, collaborative, and nonproprietary" [Benkler, 2006].

- "The opportunity to generate vibrant customer ecosystems where users help advance, implement, and even market new product features represents a largely untapped frontier for farsighted companies to exploit" [Tapscott \& Williams, 2006].

The technological foundations to make these objectives a reality are provided by a powerful infrastructure for collaborative efforts (the Internet allows people to share their efforts) and by the increased digital fluency [National-Research-Council, 1999] of the population in general, which will make owners of problems independent of “high-tech scribes' in personally meaningful tasks [Fischer, 2002]. Emerging success models, such as open source software and Wikipedia, have provided evidence of the great potential of socio-technical environments in which users can be active contributors.

\subsection{Design Time and Use Time}

In all design processes two basic stages can be differentiated: design time and use time (see Figure 1). At design time, system developers (with or without user participation) create environments and tools for the world as imagined by them to 
anticipate users' needs and objectives. At use time, users can use the system, but because their needs, objectives, and situational contexts can only be anticipated at design time, the system often requires modification to fit the users' needs [Henderson \& Kyng, 1991].

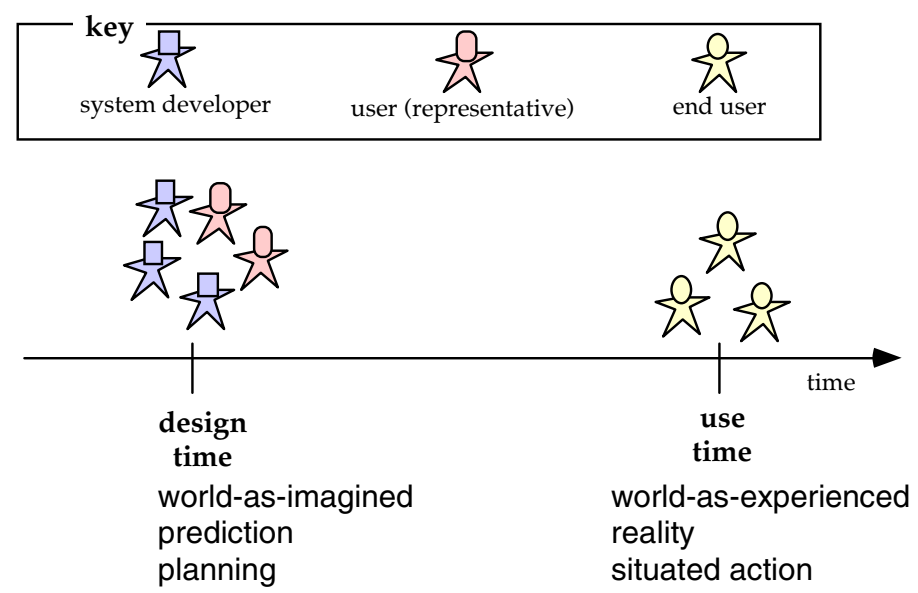

Fig. 1. Design Time and Use Time

To accommodate unexpected issues at use time, systems need to be underdesigned at design time. Underdesign [Brand, 1995] in this context does not mean less work and fewer demands for the design team, but it is fundamentally different from creating complete systems. The primary challenge of underdesign is to develop not solutions but environments that allow the "owners of problems" [Fischer, 2002] to create the solutions themselves at use time. This can be done by providing a context and a background against which situated cases, coming up during use time, can be interpreted. Underdesign is a defining activity for meta-design aimed at creating design spaces for others. It assumes that the meaning, functionality, and content of a system are not fully defined by designers and user-representatives alone at design time, but are socially constructed throughout the entire design, deployment, and use cycles of the system.

Meta-Design. By focusing equally on design and use-time activities, meta-design is different from other design methodologies such as user-centered design and participatory design. It contributes to the invention and design of cultures in which humans can express themselves and engage in personally meaningful activities. The conceptual frameworks that we have developed around meta-design explore fundamental challenges associated with design for change [Fischer \& Giaccardi, 2006]:

- How can we support skilled domain workers who are neither novices nor naive users, but who are interested in their work and who see the computer as a device to achieve their goals? 
- How can we create co-adaptive environments, in which users change because they learn, and in which systems change because users become co-developers and active contributors?

- How can we provide users with opportunities, tools, and social reward structures to extend systems to fit their needs?

Meta-design has shifted some control from designers to users and empowered users to create and contribute their own visions and objectives. Meta-design is a useful perspective for projects for which "designing the design process" is a first-class activity. This means that creating the technical and social conditions for broad participation in design activities is as important as creating the artifact itself [Wright et al., 2002].

Participatory Design for Meta-Design. Meta-design creates new demands for participatory design processes at design time by requiring: (1) the creation of systems that do not consist of a set of predetermined possibilities and functions but are designed for evolution that is being carried out by their users; and (2) a shift of focus from designing a complete system to designing a seed and mechanism for evolutionary growth and reseeding by providing content and a context for transcending the initial content.

The goal of making systems modifiable and evolvable by users does not imply transferring the responsibility of good system design to the user. Users (often being domain experts who see software development as a means to an end) will design tools and create contents of a different quality than professional software designers (for whom software is both a means and an end). Domain experts are not concerned with the tool per se, but in doing their work. However, if the tool created by the developers does not satisfy their needs or tastes, they should be able to adapt the system without always requiring the assistance of the developers. This leads to a new distribution of control for socio-technical environments.

Who Are Meta-Designers and What Do They Do? Meta-designers use their own creativity to create socio-technical environments in which other people can be creative. They must create the social conditions for broad participation in design activities which is as important as creating the artifact itself. They must encourage and facilitate the objective to develop maximum participation by activating as much knowledge as possible. The main activity of meta-designers shifts from determining the meaning, functionality, and content of a system to encouraging and supporting users to engage in these activities. Meta-designers must be willing to share control of how systems will be used, which content will be contained, and which functionality will be supported.

\section{A Process Model in Support of Meta-Design: Seeding, Evolutionary Growth,} Reseeding. The seeding, evolutionary growth, and reseeding (SER) model [Fischer \& Ostwald, 2002] is an emerging descriptive and prescriptive model for creating software systems that best fit an emerging and evolving context. In the past, large and complex software systems were built as complete artifacts through the large efforts of 
a small number of people. Instead of attempting to build complete systems, the SER model advocates building seeds that can evolve over time through the small contributions of a large number of people. It postulates that systems that evolve over a sustained time span must continually alternate between periods of planned activity and unplanned evolution, and periods of deliberate (re)structuring and enhancement. A seed is something that has the potential to change and grow. In socio-technical environments, seeds need to be designed and created for the technical as well as the social component of the environment.

\section{Examples of Socio-technical Environments Framed by Meta-design}

This section illustrates with two specific examples (Web2Gether and MAPS) how meta-design expands boundaries and redistributes control in design.

\subsection{Web2Gether: Supporting a Community of Assistive Technology Teachers}

Web2Gether [dePaula, 2004] is a socio-technical environment embedded in the larger research project of understanding and providing social and technical means to support the use of technologies in special education [Carmien et al., 2005]. Our research activities first identified the lack of social support as one of the major barriers to the adequate use of technologies in this environment. This led to a shift in our approach from a simple technical solution toward a socio-technical approach that offered means for participants to reach each other, to create and develop social networks, and to share their experiences. Web2Gether transcends an access model of technology and supports a meta-design approach in which participants can act as active contributors. It allows users to share stories and personal experiences regarding unique cases for which they came up with effective solutions to address specific needs [dePaula \& Fischer, 2005].

The success of meta-design approaches hinges on the participation of the users, requiring a deep understanding of the dynamic and transformative process of appropriation. In Web2Gether, the social and technical context were constantly negotiated and shaped by the various social groups participating at design, deployment, and use times. Figure 2 illustrates the complex environment in which Web2Gether operated as a socio-technical environment, including (1) technical components (in the lowest layer, labeled "Design Arena"); (2) work environments (with different stakeholders); and (3) the influences of institutional and national concerns, rules, and regulations. At any point of time, a change in any of these layers would require that the system be modified. Most of these changes will be experienced by the users (the community of assistive technology teachers), who need the means and knowledge to adapt and evolve the system accordingly. Our experience with Web2Gether demonstrates that the common assumption that technology's meaning, functionality, and content was set by the designers is misleading: the system was shaped by the consequences of a process of negotiation among designers, users, and other institutions. 


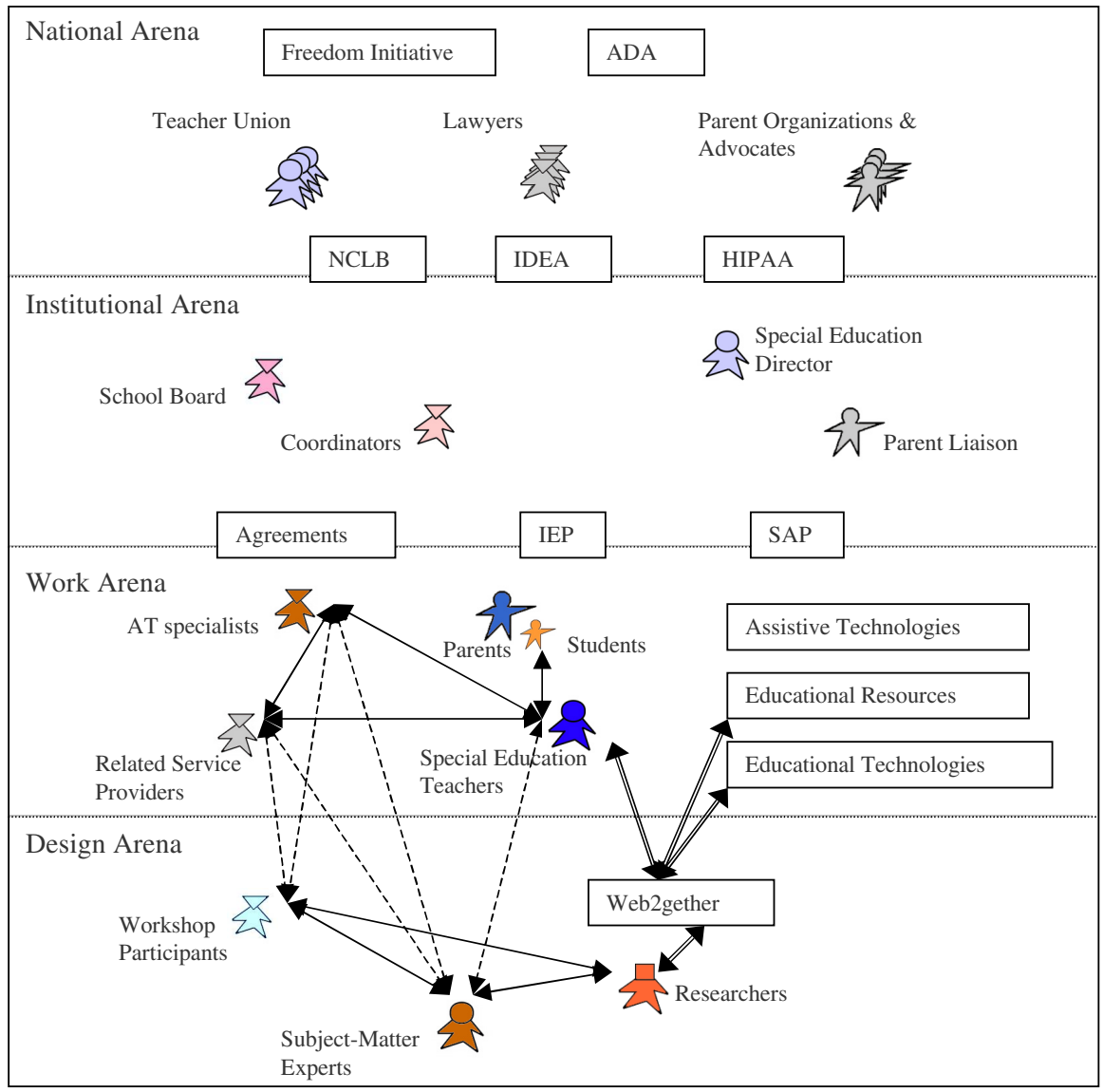

Fig. 2. Web2Gether as a Socio-Technical Environment [dePaula, 2004]

(The abbreviations used in this diagram represent the following concepts: (1) ADA: Americans with Disabilities Act; (2) NCLB: No Child Left Behind Act; (3) IDEA: Individuals With Disabilities Education Act; (4) HIPAA: Health Insurance Portability and Accountability Act; (5) IEP: Individualized Education Plan; (6) SAP: Student Disability Services; and (7) AT: Assistive Technologies)

Web2Gether was originally designed with a rudimentary understanding of a metadesign framework. It has substantially expanded our evolving framework about metadesign in the following ways [dePaula \& Fischer, 2005]:

- Participatory design: Web2Gether was socially constructed by different social groups who participated throughout this research.

- Design through cycles of closure and opening: Web2Gether went through various stages at which its concept was defined followed by changes due to new interactions between the context and social groups. 
- Co-evolution of design and context: Web2Gether was shaped to fit the needs of the context, and at the same time the context was reevaluated.

- The seeding process: This was a scaffolding process not restricted to creating initial content, but supporting structured activities, the technology, and the envisioned use community.

- Important factors: Incentive structures, integration of innovations into the practices of users, and the merging of new with existing organizational structures were critically important.

Web2Gether is a socio-technical environment in which "various possible meanings of the usefulness of a technology coexist and are constantly being negotiated and coconstructed by the groups involved in the design, implementation, deployment, and use of a technology" [dePaula, 2004]. These meanings cannot be defined once and for all at design time (see Figure 1) but require a meta-design approach in which all stakeholders can renegotiate how use contexts and technologies constitute one another and need to be open for co-evolution.

\subsection{Memory Aiding Prompting System (MAPS): An End-User Development Environment}

When users' needs are heterogeneous and their specific and exact nature is known only at use time, the strategy of "one size fits all" will not work. In our research with people with cognitive disabilities [CLever, 2005], the user populations can be characterized as a "universe of one" [Carmien, 2006] in the sense that each user's abilities are unique. To match the user's unique needs with systems, a meta-design approach is not an option-it is a necessity. An additional requirement in this application is that the users who need to act as designers to match systems to capabilities are the caregivers (e.g., parents and teachers), who generally are not computer scientists and have no interest in computers per se.

The specific problem addressed by MAPS [Carmien, 2006; Carmien et al., 2005] is how to create external scripts for a variety of tasks (including how to use public transportation systems, shopping lists, and recipes for cooking). The MAPS approach focuses on finding new ways to support distributed intelligence by complementing internal scripts (which people have in their head and which may be severely limited for people with cognitive disabilities) with external scripts supporting specific users in specific tasks.

An essential component of MAPS is an end-user development environment [Lieberman et al., 2006] supporting the creation of external scripts. Scripts consist of memory prompts with task-specific visual and auditory stimuli and feedback. In MAPS, scripts are organized as finite state sequences with state changes triggered by each unique user's actions or external events in the user's environment. One key design parameter in a scripting sequence is the granularity and specificity of a particular prompt and feedback sequence. 


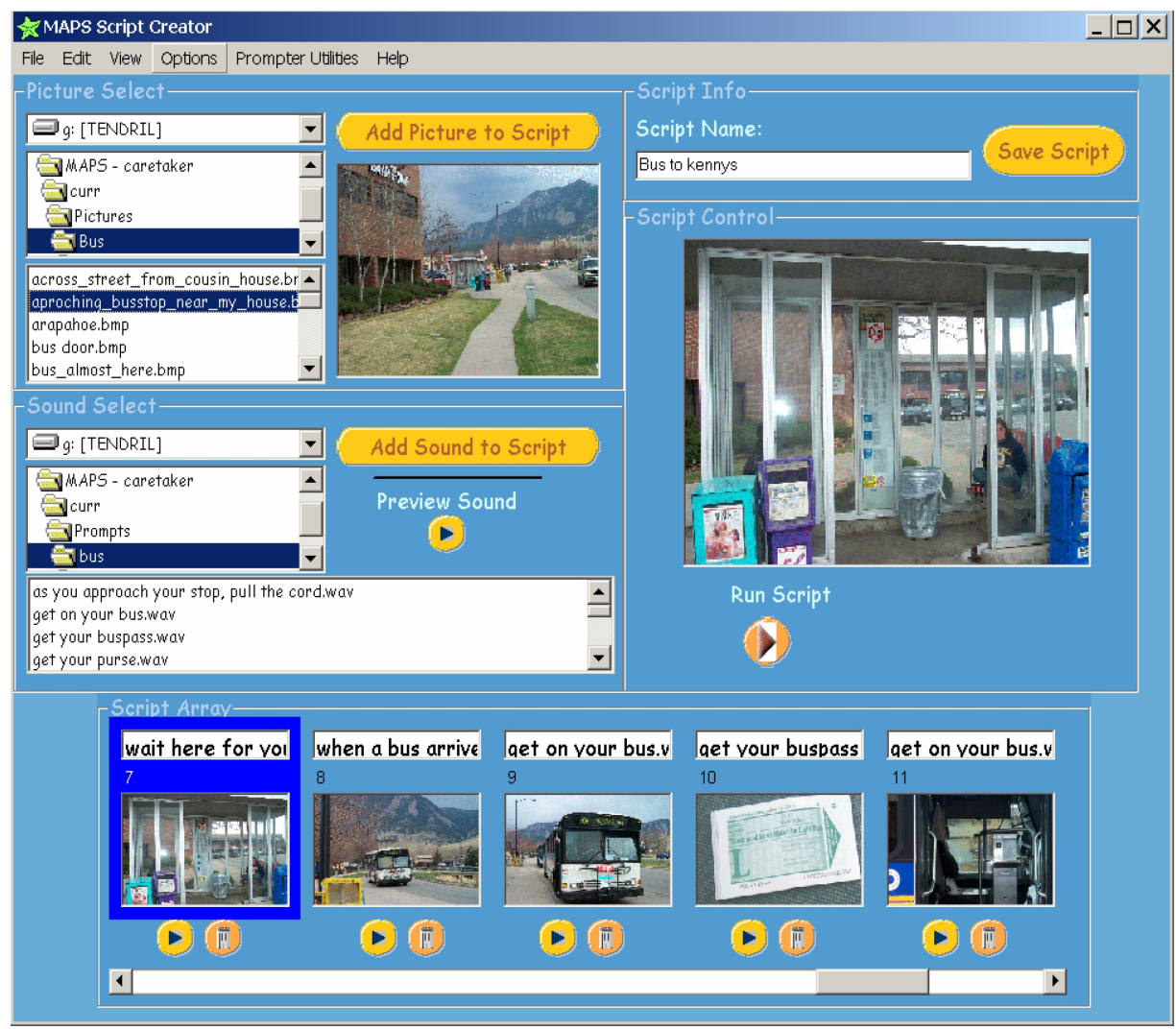

Fig. 3. An End-User Development Environment for Creating External Scripts

The scripts needed to effectively support users are specific for particular tasks, creating the requirement that the people who know about these tasks (i.e.: the local caregivers rather than technologists far removed from the action) must be able to develop scripts. In general, caregivers have no specific professional technology training, nor are they interested in becoming computer programmers. This creates the need for design environments with extensive end-user support to allow caregivers to create, store, and share scripts. Figure 3 shows the prototype of a caregiver configuration environment (embedded in MAPS) for creating complex, locationaware, multi-modal prompting sequences to support people with cognitive disabilities in using public transportation. The environment allows caregivers to assemble sound and pictures by using a filmstrip-like scripting metaphor.

\section{Assessment and Implications}

The goal of making systems modifiable and evolvable by users does not imply transferring the responsibility of good system design to the user. Socio-technical environments redistribute control in design by sharing it between developers and users. 
Expanding Boundaries. The research described in this paper has expanded a number of different boundaries. It facilitated the collaboration of stakeholders with differing background knowledge, including software engineers, HCI designers, caregivers, and people with cognitive disabilities. It broke down the sharp distinction between designers and users, and allowed users to become co-designers. It eliminated the limitations associated with closed systems by designing socio-technical environments as living entities. It allowed all stakeholders to contribute and make their voices heard. The process was supported by the seeding, evolutionary growth, and reseeding (SER) model. It explored HCI issues by exploring and addressing the unique challenges associated with universal usability and design-for-all [Newell \& Gregor, 1997]. Meta-design plays a significant role in several stages of design, including initial concept development, system configuration, integration with the use context, and specifically supporting design-in-use.

Redistributing Control. In meta-design, developers must accept a role in which they create mechanisms allowing users to act as designers and modify systems, thereby providing them with new levels of personal control. One of the major findings in our research activities was that users do not always accept and exercise these opportunities. This control is desired by users only in the case of personally meaningful problems [Fischer, 2002]. One of the pitfalls of a "do-it-yourself" society is that it can put a big burden on users (e.g., companies offloading work to customers) who may lack the experience, support, daily exposure, and interest in accomplishing these tasks. The trade-offs associated with introducing new divisions of labor [Levy \& Murnane, 2004] have to be carefully evaluated.

Our experiences gathered in the context of the design, development, and assessment of the two systems described in the previous sections indicate that metadesign methodologies do not work when users are brought into the process late, thereby denying them ownership, nor when users are "misused" to fix problems and to address weaknesses of systems that the developers did not fix themselves. It does work when users are part of the participatory design effort in establishing a metadesign framework, including support for intrinsic and extrinsic motivation, user toolkits for reducing the effort to make contributions, and the seeding of use communities in which individuals can share their contributions [Dawe, 2007].

Contributions to Socially Responsible Design. Meta-design and socio-technical environments designed as living entities contribute to socially responsible design [CPSR, 2007] in the following dimensions:

- Improving the quality of life [Carmien et al., 2005; Newell \& Gregor, 1997]: The two socio-technical environments (Web2Gether and MAPS) briefly described in this paper as part of our CLever project are developments that make an attempt to improve the quality of life for people with cognitive disabilities.

- Democratizing innovation [von Hippel, 2005]: meta-design allows owners of problems to engage in activities as a process of creating new possibilities and new artifacts, eliminating the constraint that users are restricted to what is given to them. 
- Making all voices heard [Fischer \& Giaccardi, 2006]: Complex design problems seldom fall within the boundaries of one specific domain; they require the participation and contributions of different stakeholders with various backgrounds.

- Changing professional practice [Illich, 1973]: Meta-design contributes to the creation of convivial tools which "give each person who uses them the greatest opportunity to enrich the environment with the fruits of his or her vision."

- Revolutionizing the creation of systems [Raymond \& Young, 2001; Wikipedia, 2006]: Open source software systems and collaborative content creation harness the possibilities of Web 2.0 architectures [O'Reilly, 2006], which allow that social intelligence becomes alive. Rather than relying on major contributions by a small number of people, Web 2.0 architectures derive their value from a large number of people making small contributions.

- Establishing new paradigms in learning and teaching [dePaula et al., 2001]: The impact of meta-design on teaching and learning challenges one of the most impoverished paradigms of education in which "a single, all-knowing teacher tells or shows presumably unknowing learners something they presumably know nothing about" [Bruner, 1996]. Courses-as-seeds [dePaula et al., 2001] is an educational model that explores meta-design in the context of university courses by creating a culture of informed participation [Brown \& Duguid, 2000b] by supporting community-based learning theories [Rogoff et al., 1998] with innovative collaborative technologies.

\section{Conclusion}

Meta-design expands boundaries and redistributes control in design by inventing, designing, and supporting a culture in which all stakeholders in socio-technical environments can express themselves and engage in personally meaningful activities. Meta-design requires a new mindset of all participants. Specifically, developers must give up some control at design time and users must be willing to act as active contributors and not just passive consumers at use time. Meta-design raises many issues and research problems of fundamental importance, including new design methodologies; new understandings of cognition, collaboration, and motivation; and the design of new media and new technologies.

Acknowledgements. The author thanks the members of the Center for LifeLong Learning \& Design at the University of Colorado, who have made major contributions to ideas described in this paper. The $\mathrm{PhD}$ research of Stefan Carmien, Rogerio dePaula, and Melissa Dawe has specifically influenced our research about socially responsible interaction.

The research was supported by grants from (1) the National Science Foundation, (a) REC-0106976 "Social Creativity and Meta-Design in Lifelong Learning Communities", (b) IIS-0613638 "A Meta-Design Framework for Participative Software Systems", (c) IIS- 0709304 "A New Generation Wiki for Supporting a 
Research Community in Creativity and IT"; (2) SRA Key Technology Laboratory, Inc., Tokyo, Japan; and (3) the Coleman Institute, Boulder, CO.

\section{References}

1. Alexander, C.: The State of the Art in Design Methods. In: Cross, N. (ed.) Developments in Design Methodology, pp. 309-316. John Wiley \& Sons, New York (1984)

2. Benkler, Y.: The Wealth of Networks: How Social Production Transforms Markets and Freedom. Yale University Press, New Haven (2006)

3. Brand, S.: How Buildings Learn: What Happens After They're Built. Penguin Books, New York (1995)

4. Brown, J.S., Duguid, P.: Re-education. In: Brown, J.S., Duguid, P. (eds.) The Social Life of Information, pp. 207-241. Harvard Business School Press, Boston, MA (2000a)

5. Brown, J.S., Duguid, P.: The Social Life of Information. Harvard Business School Press, Boston, MA (2000b)

6. Bruner, J.: The Culture of Education. Harvard University Press, Cambridge, MA (1996)

7. Carmien, S.: Socio-Technical Environments Supporting Distributed Cognition for Persons with Cognitive Disabilities, Ph.D. Dissertation, University of Colorado at Boulder (2006), Available at: http://13d.cs.colorado.edu/ carmien/

8. Carmien, S., Dawe, M., Fischer, G., Gorman, A., Kintsch, A., Sullivan, J.F.: SocioTechnical Environments Supporting People with Cognitive Disabilities Using Public Transportation. Transactions on Human-Computer Interaction (ToCHI) 12(2), 233-262 (2005)

9. CLever: Cognitive Levers - Helping People Help Themselves, Available (2005)

10. CPSR: Computer Professionals for Social Responsibility (2007), Available at http://www.cpsr.org/

11. Dawe, M.: Reflective Design-In-Use: Co-Designing an Assistive Remote Communication System with Individuals with Cognitive Disabilities and their Families, Ph.D. Dissertation, University of Colorado at Boulder (2007), Available at http://13d.cs.colorado.edu/ meliss/ diss/

12. de Paula, R.: The Construction of Usefulness: How Users and Context Create Meaning with a Social Networking System, Ph.D. Dissertation, University of Colorado at Boulder (2004)

13. de Paula, R., Fischer, G.: Knowledge Management: Why Learning from the Past is not Enough! In: Davis, J., Subrahmanian, E., Westerberg, A. (eds.) Knowledge Management: Organizational and Technological Dimensions, pp. 21-54. Physica Verlag, Heidelberg (2005)

14. de Paula, R., Fischer, G., Ostwald, J.: Courses as Seeds: Expectations and Realities. In: Dillenbourg, P., Eurelings, A., Hakkarainen, K. (eds.) Proceedings of The European Conference on Computer-Supported Collaborative Learning, Maastricht, Netherlands, pp. 494-501 (2001)

15. Fischer, G.: Beyond 'Couch Potatoes': From Consumers to Designers and Active Contributors, in FirstMonday (Peer-Reviewed Journal on the Internet) (2002), Available at http://firstmonday.org/issues/issue7_12/fischer/

16. Fischer, G., Giaccardi, E.: Meta-Design: A Framework for the Future of End User Development. In: Lieberman, H., Paternò, F., Wulf, V. (eds.) End User Development: Empowering People to Flexibly Employ Advanced Information and Communication Technology, pp. 427-457. Kluwer Academic Publishers, Dordrecht, The Netherlands (2006) 
17. Fischer, G., Giaccardi, E., Ye, Y., Sutcliffe, A.G., Mehandjiev, N.: Meta-Design: A Manifesto for End-User Development. Communication of the ACM 47(9), 33-37 (2004)

18. Fischer, G., Ostwald, J.: Seeding, Evolutionary Growth, and Reseeding: Enriching Participatory Design with Informed Participation, pp. 135-143. Malmö University, Sweden (2002)

19. Giaccardi, E.: Principles of Metadesign: Processes and Levels of Co-Creation in the New Design Space; Ph.D. Dissertation, CAiiA-STAR, School of Computing, Plymouth, UK (2004)

20. Henderson, A., Kyng, M.: There's No Place Like Home: Continuing Design in Use. In: Greenbaum, J., Kyng, M. (eds.) Design at Work: Cooperative Design of Computer Systems, pp. 219-240. Lawrence Erlbaum Associates, Inc, Hillsdale, NJ (1991)

21. Illich, I.: Tools for Conviviality. Harper and Row, New York (1973)

22. Levy, F., Murnane, R.J.: The New Division of Labor: How Computers are Creating the Next Job Market. Princeton University Press, Princeton (2004)

23. Lieberman, H., Paterno, F., Wulf, V. (eds.): End User Development - Empowering people to flexibly employ advanced information and communication technology. Kluwer Publishers, Dordrecht, The Netherlands (2006)

24. Mumford, E.: Sociotechnical Systems Design: Evolving Theory and Practice. In: Bjerknes, G., Ehn, P., Kyng, M. (eds.) Computers and Democracy, Avebury, Aldershot, UK, pp. 5976 (1987)

25. Nardi, B.A.: A Small Matter of Programming. The MIT Press, Cambridge, MA (1993)

26. National-Research-Council: Being Fluent with Information Technology, National Academy Press, Washington, DC (1999)

27. National-Research-Council: Beyond Productivity: Information Technology, Innovation, and Creativity, National Academy Press, Washington, DC (2003)

28. Newell, A.F., Gregor, P.: Human Computer Interfaces for People with Disabilities. In: Helander, M.G., Landauer, T.K., Prabhu, P.V. (eds.) Handbook of Human-Computer Interaction, vol. 1, pp. 813-824. Elsevier Science B.V, Amsterdam (1997)

29. O'Reilly, T.: What Is Web 2.0 - Design Patterns and Business Models for the Next Generation of Software (2006), Available at http://www.oreillynet.com/pub/a/oreilly/ tim/news/2005/09/30/what-is-web-20.html

30. Raymond, E.S., Young, B.: The Cathedral and the Bazaar: Musings on Linux and Open Source by an Accidental Revolutionary. O'Reilly \& Associates, Sebastopol, CA (2001)

31. Rittel, H.: Second-Generation Design Methods. In: Cross, N. (ed.) Developments in Design Methodology, pp. 317-327. John Wiley \& Sons, New York (1984)

32. Rogoff, B., Matsuov, E., White, C.: Models of Teaching and Learning: Participation in a Community of Learners. In: Olsen, D.R., Torrance, N. (eds.) The Handbook of Education and Human Development: New Models of Learning, Teaching and Schooling, pp. 388414. Blackwell, Oxford (1998)

33. Scaffidi, C., Shaw, M., Myers, B.: Estimating the Numbers of End Users and End User Programmers. In: VL/HCC'05. Proceedings of 2005 IEEE Symposium on Visual Languages and Human-Centric Computing, Dallas,Texas, September, pp. 207-214 (2005)

34. Suchman, L.: Plans and Situated Actions: The Problem of Human-Machine Communication. Cambridge University Press, Cambridge, England (1987)

35. Tapscott, D., Williams, A.D.: Wikinomics: How Mass Collaboration Changes Everything, Portofolio, Penguin Group, New York, NY (2006)

36. Terveen, L.G.: An Overview of Human-Computer Collaboration. Knowledge-Based Systems Journal, Special Issue on Human-Computer Collaboration 8(2-3), 67-81 (1995) 
37. Trist, E.L.: The Sociotechnical Perspective: The Evolution of Sociotechnical Systems as a Conceptual Framework and as an Action Research Program. In: VanDeVen, A.H., Joyce, W.F. (eds.) Perspectives on Organization Design and Behavior, pp. 19-75. Wiley, New York, NY (1981)

38. von Hippel, E.: Democratizing Innovation. MIT Press, Cambridge, MA (2005)

39. Wikipedia: The Free Encyclopedia (2006), Available at http://wikipedia.org/

40. Winograd, T., Flores, F.: Understanding Computers and Cognition: A New Foundation for Design. Ablex Publishing Corporation, Norwood, NJ (1986)

41. Wright, M., Marlino, M., Sumner, T.: Meta-Design of a Community Digital Library, DLib Magazine, vol. 8(5), Available (2002), at http://www.dlib.org/dlib/may02/wright/ 05wright.html 